\title{
OSCILLATION AND NONOSCILLATION IN NONLINEAR THIRD ORDER DIFFERENCE EQUATIONS
}

\author{
B. SMITH and W.E. TAYLOR, JR. \\ Department of Mathematics \\ Texas Southern University \\ Houston, TX 77004 \\ (Received August 18, 1988 and in revised form June 20, 1989)
}

KEY WORDS AND PHRASES. Difference equations, third order, oscillatory and nonoscillatory solutions. 1980 AMS SUBJECT CLASSIFICATION CODES. 39A10, 39A12.

1. INTRODUCTION.

This paper is concerned with the oscillatory behavior of solutions of the third order nonlinear difference equation

$$
\Delta\left(P_{n} \Delta^{2} v_{n}\right)+Q_{n} f\left(v_{n+1}, \Delta v_{n+1}, \Delta^{2} v_{n+1}\right)=0, n=1,2, \ldots,
$$

where $\Delta$ is the forward difference operator 1.e., $\Delta v_{n}=v_{n+1}-v_{n}$. It will be assumed throughout that the conditions below are satisfied:

$$
\begin{aligned}
& \text { (I) } P_{n}>0, \Delta P_{n}>0 \text { and } Q_{n}>0 \text { for } n=0,1,2, \ldots \\
& \text { (II) } \sum_{n}^{\infty} \frac{1}{P_{n}}=\infty \\
& \text { (III) } f: R^{3}+R \text { is continuous and } x f(x, y, z)>0 \text { for } x \neq 0 .
\end{aligned}
$$

By a solution of (1.1) we mean a real sequence $V$ satisfying equation (1.1) for $n=1,2, \ldots$. A solution $V$ of (1.1) is called nonoscillatory if it is eventually positive or eventually negative. Otherwise, it is called oscillatory. The problem of determining oscillation criteria for certain second order nonlinear difference equations has been investigated by Hooker and Patula [1], and Szmanda [2]. The results of [2] were generalized by Li [3]. This paper examines a slightly more general equation than those studied in [2] and [3]. The authors began a study of similar equations in [4]. 
2. MAIN RESULTS.

LEMMA 2.1. Suppose $V$ is a nonoscillatory solution of (1.1). Then, efther

$$
\operatorname{sgn} v_{n}=\operatorname{sgn} \Delta v_{n}=\operatorname{sgn} \Delta^{2} v_{n}
$$

for all n sufficiently large, or

$$
\operatorname{sgnv} v_{n}=\operatorname{sgn} \Delta^{2} v_{n} \neq \operatorname{sgn} \Delta v_{n}
$$

for all sufficiently large $n$, and $\underset{n \rightarrow \infty}{\lim } \underset{n}{\Delta v_{n}=\lim _{n \rightarrow \infty}} \Delta^{2} v_{n}=0$.

PROOF. Assume $V$ is a nonoscillatory solution of (1.1), where $v_{n}>0$ for all $\mathrm{n}>\mathrm{N}$, where $\mathrm{N}$ is a positive integer. The proof is simflar if $v_{n}\langle 0$ for all $n\rangle N$. Note that $\Delta\left(P_{n} \Delta^{2} V_{n}\right)=-Q_{n} f\left(V_{n+1}, \Delta V_{n+1}, \Delta^{2} V_{n+1}\right)<0$, for each $n>N$. Thus $P_{n} \Delta^{2} V_{n} 1 s$ decreasing and is eventually sign definite. A positive integer $M>N$ exists for which $\Delta v_{n}$ and $\Delta^{2} v_{n}$ are of one sign when $n>M$. The following cases must be considered:

(a) ${ }^{n} v_{n}>0, \Delta v_{n}>0, \Delta^{2} v_{n}>0, n>M$,

(b) $v_{n}>0, \Delta v_{n}<0, \Delta^{2} v_{n}>0, n>M$,

(c) $v_{n}>0, \Delta v_{n}<0, \Delta^{2} v_{n}<0, n>M$,

(d) $v_{n}>0, \Delta v_{n}>0, \Delta^{2} v_{n}<0, n>M$.

Case (c) is impossible because if $\Delta V_{n} \Delta^{2} v_{n}>0$ for all sufficlently large $n$, then $\operatorname{sgnV} V_{n}=\operatorname{sgn} \Delta V_{n}$ eventually. We show that (d) is also impossible. If (d) holds, then from above $P_{n} \Delta^{2} V_{n}$ is negative and decreasing for all $n$ sufficiently large. Let $k<0$ be such that $P_{n} \Delta^{2} v_{n}<k$ for all $n>M$. Then $\Delta^{2} v_{n}<\frac{k}{P_{n}}, n>M$. Summing from $M$ to $R-1$ we obtain

$$
\Delta V_{R}-\Delta V_{M}<k \sum_{n=m}^{R-1} \frac{1}{P_{n}} .
$$

Letting $R \rightarrow \infty$, implies $\Delta V_{R}$ is eventually negative, but this contradicts (d), therefore (d) cannot hold. This completes the proof of the lemma.

We continue our study of $(1.1)$ by considering a functional which plays a vital role in our investigation. Similar functionals have been used to study solutions of differential equations (Taylor [5]).

LEMMA 2.2. Let $\mathrm{V}$ be a solution of (1.1). Then

$$
F\left[V_{n}\right]=F_{n}=2 V_{n} P_{n} \Delta^{2} V_{n}-P_{n-1}\left(\Delta V_{n}\right)^{2}
$$


is nonincreasing, in fact

$$
\Delta F_{n}=-2 Q_{n} V_{n+1} f\left(V_{n+1}, \Delta V_{n+1}, \Delta^{2} v_{n+1}\right)-P_{n}\left(\Delta^{2} v_{n}\right)^{2}-\Delta P_{n-1}\left(\Delta V_{n}\right)^{2}
$$

Since $F_{n}$ is monotone for any nontrivial solution of (1.1) we have that $F_{n}$ is of one sign for all n sufficiently large. Using this we will examine solutions of (1.1) where $F_{n}>0$ for each $n$ and those for which $F_{m}<0$ for some positive integer $m$.

THEOREM 2.1. Let $V$ be a nontrivial solution of $(1.1)$ for which $F\left[V_{n}\right]>0$. Then the following are true:

$$
\sum^{\infty} Q_{n} v_{n+1} f\left(v_{n+1}, \Delta v_{n+1}, \Delta^{2} v_{n+1}\right)<\infty,
$$

$$
\sum_{n}^{\infty} P_{n}\left(\Delta^{2} v_{n}\right)^{2}<\infty \text {, and }
$$

$$
\sum_{n-1}^{\infty}\left(\Delta V_{n}\right)^{2}<\infty
$$

PROOF. Since $F_{n}>0$ for each $n$, differencing $F_{n}$ and summing from 0 to $k-1$ we find

$$
\begin{aligned}
0<F_{k}= & F_{0}-2 \sum_{0}^{k-1} Q_{n} v_{n+1} f\left(v_{n+1}, \Delta v_{n+1}, \Delta^{2} v_{n+1}\right)- \\
& \sum_{0}^{k-1} P_{n}\left(\Delta^{2} v_{n}\right)^{2}-\sum_{0}^{k-1} \Delta P_{n-1}\left(\Delta v_{n}\right)^{2} .
\end{aligned}
$$

Thus,

$$
\begin{aligned}
& 2 \sum_{0}^{k-1} Q_{n} v_{n+1} f\left(v_{n+1}, \Delta v_{n+1}, \Delta^{2} v_{n+1}\right)+ \\
& \sum_{0}^{k-1} P_{n}\left(\Delta^{2} v_{n}\right)^{2}+\sum_{0}^{k-1} \Delta P_{n-1}\left(\Delta V_{n}\right)^{2}<F_{0} .
\end{aligned}
$$

Allowing $k$ to tend to infinity establishes each of (1), (11) and (1i1) since $F_{0}$ is Independent of $k$.

THEOREM 2.2. Suppose that $\frac{f(x, y, z)}{x}>r>0$ for $x \neq 0$ and 1 im inf $Q_{n}>0$. Let $v$ be a solution of (1.1) for which $F\left[V_{n}\right]>0$ for each $n$. Then

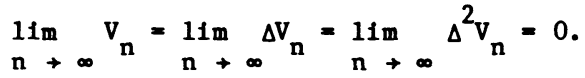

PROOF. To prove (iv), observe that

$$
v_{n+1} f\left(v_{n+1}, \Delta v_{n+1}, \Delta^{2} v_{n+1}\right)>r v_{n+1}^{2} .
$$

Thus

$$
\begin{aligned}
& Q_{n} v_{n+1} f\left(v_{n+1}, \Delta v_{n+1}, \Delta v_{n+1}\right)>\propto v_{n+1}^{2} \\
& \text { where } \alpha=\lim \text { inf } Q_{n}, \text { so we have }
\end{aligned}
$$




$$
\alpha \underset{0}{\infty} \sum_{n+1}^{\infty} v_{n}^{2}<\sum_{0}^{\infty} Q_{n} v_{n+1} f\left(v_{n+1}, \Delta v_{n+1}, \Delta^{2} v_{n+1}\right)
$$

Now apply (i), of Theorem 2.1, and the proof of (1v) is complete.

The relations ( $v$ ) follow as a consequence of (iv).

EXAMPLE 2.1. As an illustration of Theorem 2.2 consider equation (1.1) with $P_{n}=n$,

$$
Q_{n}=\frac{2^{2 n}(n-1)}{2^{2 n+2}+1}, f(x, y, z)=x^{3}+x
$$

Then

$$
\Delta\left(n \Delta^{2} v_{n}\right)+\frac{2^{2 n}(n-1)}{2^{2 n+2}+1}\left(v_{n+1}^{3}+v_{n+1}\right)=0
$$

The sequence defined by $2^{-n}$ is a solution of this equation for which $F_{n}+0$ as $n \rightarrow \infty$.

THEOREM 2.3. If $\frac{f(x, y, z)}{x}>r>0$, and $\sum_{n}^{\infty} Q_{n}=\infty$, then every nonoscillatory solution of (1.1) approaches zero as $n \rightarrow \infty$.

PROOF. Suppose $V$ is an eventually positive solution of (1.1) that is bounded away from zero, 1.e. $v_{n}>B>0$ for all $n$ sufficiently large. Because of Lemma 2.1 , an integer $M$ exists so that the relations $(2.1)$ or (2.2) are satisfied by $V$ for all $n>M . \quad$ Now $f\left(v_{n+1}, \Delta v_{n+1}, \Delta^{2} v_{n+1}\right)>r v_{n+1}$ where $r$ is a positive constant. From (1.1) we find

Thus,

$$
\Delta\left(P_{n} \Delta^{2} v_{n}\right)=-Q_{n} f\left(V_{n+1}, \Delta V_{n+1}, \Delta^{2} v_{n+1}\right)
$$

$$
\Delta\left(P_{n} \Delta^{2} v_{n}\right)<-r Q_{n} v_{n+1}
$$

Summing both sides of (2.3) from $M$ to $k-1$ we find

$$
P_{k} \Delta^{2} v_{k}<P_{M} \Delta^{2} v_{M}-r \sum Q_{n}^{k-1} v_{n+1}<P_{M} \Delta^{2} v_{M}-r \beta \sum Q_{n} \cdot
$$

But as $k+\infty$, the right hand side of (2.4) tends to $-\infty$, which in turn forces $P_{k} \Delta^{2} V_{k}$ to tend to $-\infty$, and hence $\Delta^{2} v_{k}<0$ eventually, a contradiction of relations (2.1) and (2.2). A similar argument treats the case of an eventually negative solution. This completes the proof of the theorem.

COROLLARY 2.1. If $\frac{f(x, y, z)}{x}>r>0$, for $x \neq 0$, and $\sum^{\infty} Q_{n}=\infty$, then every nonoscillatory solution of (1.1) satisfies the relations (2.2).

We are now in a position to show that oscillatory solutions exist under certain conditions.

THEOREM 2.4. Suppose $\frac{f(x, y, z)}{x}>r>0$, for $x \neq 0, \sum_{n}^{\infty}=\infty$, and $P_{n}$ is bounded. If $V$ is a solution of (1.1) for which $F\left[V_{n}\right]<0$ for some $n$, then $V$ is oscillatory.

PROOF. Suppose $\mathrm{V}$ is a nonoscillatory solution of (1.1). We may suppose without any generality loss that $V_{n}>0$ and $F\left[V_{n}\right]<0$ for all $n>N$, since $F\left[V_{n}\right]$ is nonincreasing as $n+\infty$. From Theorem 2.3, $v_{n}+0, \Delta v_{n}+0$ and $\Delta^{2} v_{n}+0$ as $n+\infty$. 
This together with the boundedness of $P_{n}$ implies that $F\left[V_{n}\right]+0$. This is clearly impossible since $F_{n}<0$ and $\Delta F_{n}<0$ for large $n$ and the proof follows by contradiction.

Under certain conditions the bounded solutions of (1.1) behave rather nicely. Similar results appeared in [2] and [3].

THEOREM 2.5. Suppose $\sum^{\infty} n Q_{n}=\infty$ and $P_{n}<B, \beta$ constant. Then every bounded solution of (1.1) is either oscillatory or tends to zero monotonically.

PROOF. By Lemma 2.1 a bounded nonoscillatory solution $V$ satisfies

$$
\operatorname{sgnV} v_{n}=\operatorname{sgn} P_{n} \Delta^{2} v_{n} \neq \operatorname{sgn} \Delta v_{n}
$$

for all n sufficiently large. Assume that $v_{n}>0$ eventually and suppose $\lim _{n \rightarrow \infty} \Delta V_{n}=A_{0}$ where $A_{0}>0$. Note also

$$
\lim _{n \rightarrow \infty} \Delta V_{n}=\lim _{n \rightarrow \infty} P_{n} \Delta^{2} V_{n}=\lim _{n \rightarrow \infty} \Delta^{2} v_{n}=0
$$

The fact that $P_{n} \Delta^{2} v_{n} \rightarrow 0$ as $n \rightarrow \infty$ follows from the boundedness of $v_{n}$ and (II). Consider the sequence $r_{n}=n\left(P_{n} \Delta^{2} v_{n}\right)$. Note that

$$
\Delta r_{n}=P_{n+1} \Delta^{2} v_{n+1}-n Q_{n} f\left(v_{n+1}, \Delta V_{n+1}, \Delta^{2} v_{n+1}\right)
$$

Since $f$ is continuous $f\left(v_{n+1}, \Delta V_{n+1}, \Delta^{2} v_{n+1}\right)+f\left(A_{0}, 0,0\right)>0$ as $n+\infty$ so there exists $N$ such that

$$
f\left(v_{n+1}, \Delta V_{n+1}, \Delta^{2} v_{n+1}\right)>\frac{1}{2} f\left(A_{0}, 0,0\right) \text { for all } n>N \text {. }
$$

Therefore from (2.5) we have

$$
\begin{aligned}
& \Delta r_{n}<P_{n+1} \Delta^{2} v_{n+1}-\frac{1}{2} n Q_{n} f\left(A_{0}, 0,0\right) \\
& <B \Delta^{2} v_{n+1}-\frac{1}{2} n Q_{n} f\left(A_{0}, 0,0\right) .
\end{aligned}
$$

Summing, from $\mathrm{N}$ to $\mathrm{n}$

$$
r_{n+1}<r_{N}+B \Delta V_{n+2}-\beta V_{N+1}-\frac{1}{2} f\left(A_{0}, 0,0\right) \sum_{j=N}^{n} j Q_{j} \cdot
$$

As $n+\infty, r_{n} \rightarrow-\infty$, a contradiction. Therefore $A_{0}=0$. This completes the proof of the theorem.

Finally we have

THEOREM 2.6. Suppose $\sum^{\infty} Q_{n}=\infty, f(\alpha x, \alpha y, \alpha z)=\alpha^{2 m+1} f(x, y, z), \alpha \neq 0$ and $f(x, y+h, z)>f(x, y, z)$ for $h>0$. Then every solution of (1.) is either bounded or oscillatory. 
PROOF. Suppose $v_{n}$ is an unbounded nonoscillatory solution of (1.1). Without loss of generality we have

$$
\mathrm{v}_{\mathrm{n}}>0, \Delta \mathrm{v}_{\mathrm{n}}>0, \mathrm{P}_{\mathrm{n}} \Delta^{2} \mathrm{v}_{\mathrm{n}}>0
$$

for all n sufficiently large. Consider the functional

$$
q_{n}=\frac{P_{n} \Delta^{2} v_{n}}{v_{n}}
$$

Differencing $q_{n}$ we find

$$
\begin{aligned}
\Delta q_{n} & =\frac{-Q_{n} f\left(v_{n+1}, \Delta v_{n+1}, \Delta^{2} v_{n+1}\right)}{v_{n+1}}-\frac{P_{n} \Delta v_{n} \Delta^{2} v_{n}}{v_{n} v_{n+1}} \\
& <-v_{n+1}^{2 m} Q_{n} f\left(1, \frac{\Delta v_{n+1}}{v_{n+1}}, \frac{\Delta^{2} v_{n+1}}{v_{n+1}}\right) \\
& <-v_{n+1}^{2 m} Q_{n} f\left(1,0, \frac{\Delta^{2} v_{n+1}}{v_{n+1}}\right) \\
& <-\frac{v_{N}^{2 m} Q_{n} f(1,0,0)}{2} .
\end{aligned}
$$

Summing we obtain

$$
q_{m}<k_{0}-\frac{v_{N}^{2 m} f(1,0,0)}{2} \sum_{N}^{m-1} Q_{n} .
$$

But this implies $q_{m}+-\infty$ as $m+\infty$, a contradiction since $P_{n}, \Delta^{2} v_{n}$ and $v_{n}$ are positive for all n sufficiently large.

EXAMPLE 2.2. It is possible for equations of the form of (1.1) to have unbounded oscillatory solutions. The sequence $v_{n}=(-2)^{n}$ is a solution of

$$
\Delta^{3} v_{n}+\frac{11}{18\left(4^{n+1}\right)}\left(\Delta v_{n+1}\right)^{3}+3 v_{n+1}=0
$$

Note that this example does not violate the conclusion of Theorem 2.6. Note also that (III) is not satisfied.

\section{REFERENCES}

1. HOOKER, J.W. and PATULA, W.T., A Second-order Nonlinear Difference Equation: Oscillation and Asymptotic Behavior, J. Math. Anal. Appl. 91 (1983), 9-29.

2. SZMANDA, B., Oscillation Theorems for Nonlinear Second-order Difference Equations, J. Math. Ana1. App1. 79 (1981), 90-95.

3. L1, Z.H., A Note on the 0scillatory Property for Nonlinear Difference Equations and Differential Equations, J. Math. Ana1. Appl. 103 (1984), 344-352.

4. SMITH, B. and TAYLOR, W.E., Jr., Asymptotic Behavior of Solutions of a Third Order Difference Equation, Port. Math. 44 (1987), 113-117.

5. TAYLOR, W.E., Jr., Asymptotic Behavior of Solutions of a Fourth Order Nonlinear 


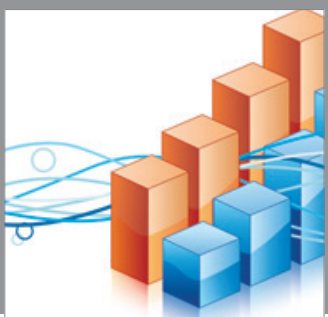

Advances in

Operations Research

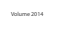

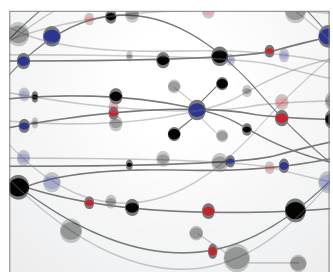

\section{The Scientific} World Journal
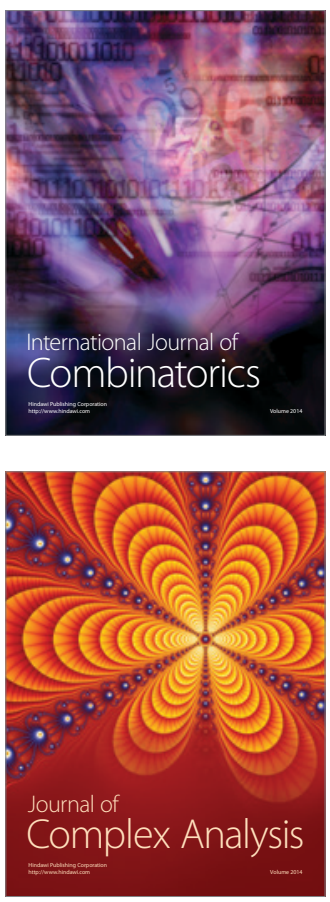

International Journal of

Mathematics and

Mathematical

Sciences
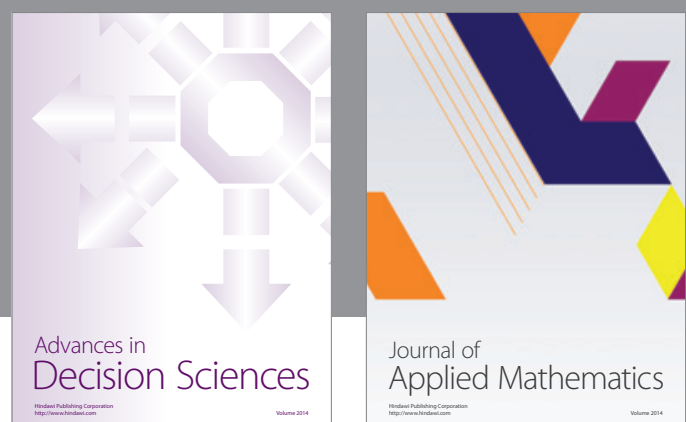

Journal of

Applied Mathematics
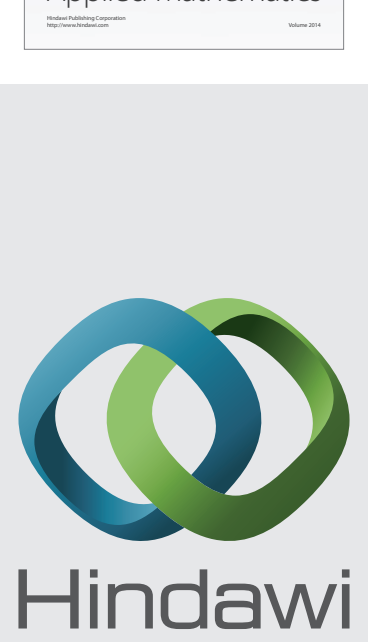

Submit your manuscripts at http://www.hindawi.com
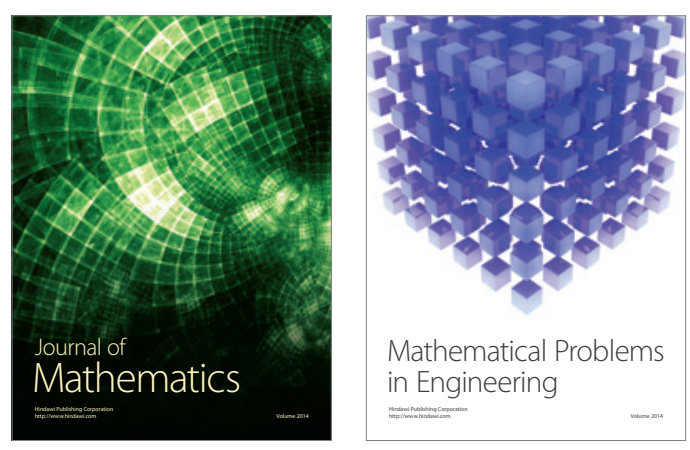

Mathematical Problems in Engineering
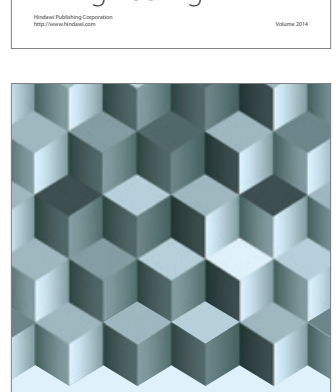

Journal of

Function Spaces
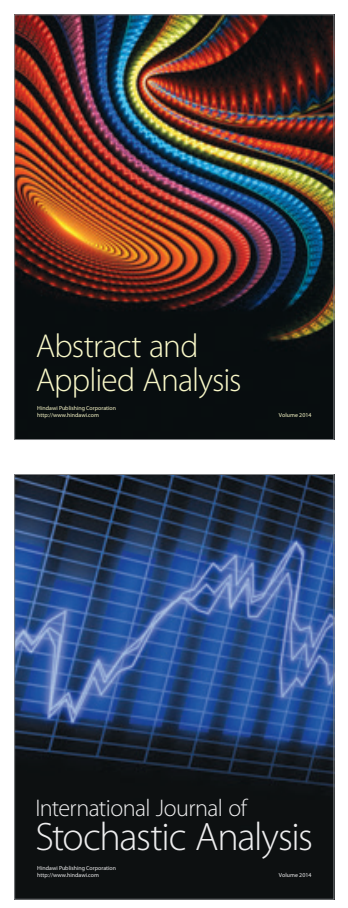

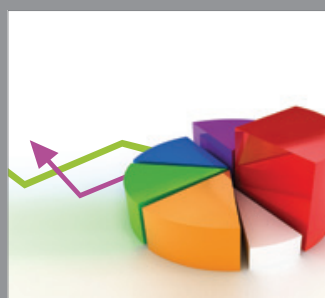

ournal of

Probability and Statistics

Promensencen
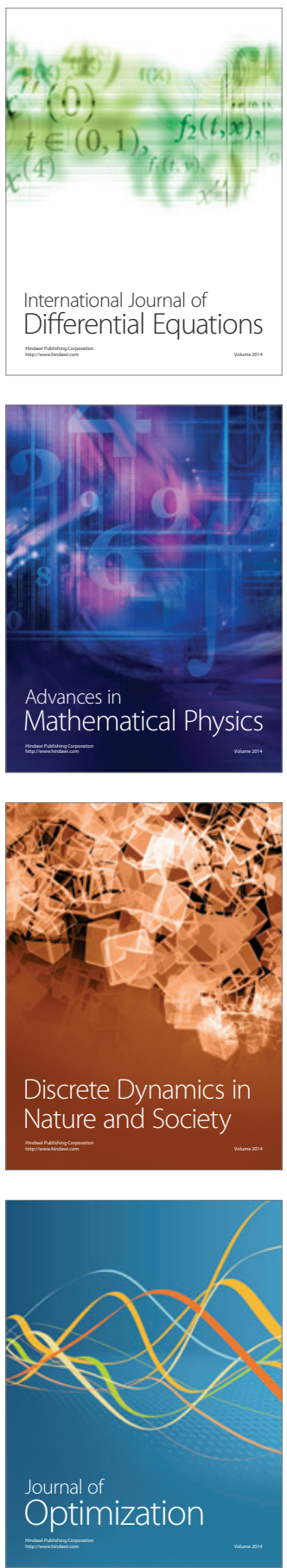